\title{
ANÁLISE DE FATORES DE RISCO ASSOCIADOS À INDICAÇÃO DE RECONSTRUÇÃO MAMÁRIA IMEDIATA
}

\author{
RISK FACTORS ANALYSIS ASSOCIATED TO IMMEDIATE MAMMARY \\ RECONSTRUCTION INDICATION
}

Maíra de Camargos Resende1, Mirna Namie Okamura², Luis Afonso de Araújo Peixoto da Costa Rodrigues ${ }^{1}$, Marcelo Moura Costa Sampaio², Alfredo Carlos Simões Dornellas Barros ${ }^{2}$, Bernardo Pinheiro da Senna Nogueira Batista².

\section{RESUMO}

OBJETIVO: Esse trabalho teve como objetivo identificar se os fatores de risco descritos estão associados à contra-indicação de reconstrução mamária imediata em um serviço filantrópico da cidade de São Paulo. MÉTODOS: Registros de uma base de dados mantida prospectivamente incluindo todas as pacientes operadas no Serviço de Filantropia do Núcleo de Mastologia do Hospital Sírio Libanês durante o ano de 2015 foi consultado. Os fatores de risco analisados foram: Idade, comorbidades, tabagismo, índice de massa corporal e tratamento neoadjuvante. A indicação de reconstrução imediata das mamas foi considerada como desfecho principal. RESULTADOS: Foram incluídas no estudo 225 pacientes operadas por câncer de mama. Destas, $124(55,1 \%)$ pacientes realizaram a reconstrução mamária no mesmo tempo cirúrgico. A idade média das pacientes que realizaram reconstrução imediata das mamas foi $52,3 \mathrm{com}$ desvio padrão de 1,0 . Cento e trinta e oito pacientes (61,3\%) possuíam alguma comorbidade clínica. Dessas, 65 (47\%) realizaram reconstrução imediata. Das pacientes tabagistas, $16(59,3 \%)$ realizaram resconstrução imediata. Já do grupo de pacientes composto por ex tabagistas e por pacientes que nunca fumaram, $102(58,7 \%)$ foram submetidas ao procedimento cirúrgico. A média de IMC das pacientes que realizaram reconstrução imediata das mamas foi 27 com desvio padrão de 0,4. Das pacientes que realizaram neoadjuvância, 55 (73,3\%), também foram submetidas à reconstrução imediata. CONCLUSÃO: A indicação cirúrgica deve ser personalizada, levando em consideração diversas características da paciente.

Descritores: neoplasias da mama, mamoplastia, fatores de risco.

\section{ABSTRACT}

OBJECTIVE: This article had as objective to identify if the risk factors described are associated to contraindication of immediate mammary reconstruction in a philanthropic service of the city of São Paulo. METHODS: Records from a database maintained prospectively including all operated patients in the Philanthropy Service of Mastology Nucleus of the Sírio Libanês Hospital during 2015 were consulted. The risk factors analysed were: Age, comorbidities, tabagism, BMI, neoadjuvant treatment. The indication of the immediate reconstruction of breasts was considerate as main resort. RESULTS: 225 breast cancer operated patients were included in the study. From these, $124(55,1 \%)$ patients realized the breasts reconstruction in the same surgical time. The average age from the patients that realized this procedure was 52,3 with a standard deviation of 1,0. 138 patients $(61,3 \%)$ had some clinical comorbidity. From these, $65(47 \%)$ realized immediate reconstruction. From the smoking patients, $16(59,3 \%)$ did the same procedure. Now, from the group made of former smoking and non-smoking, 102 $(58,7 \%)$ were submitted to the surgical procedure. The average BMI of the patients that realized immediate breasts reconstruction was 27 with a standard deviation of 0,4. From the patients that realized neoadjuvant treatment, 55 (73,3\%) were also submitted to immediate reconstruction. CONCLUSION: The surgery indication must be personalized, considering various characteristics from each patient.

Keywords: breast neoplasms, mammoplasty, risk factors.

\footnotetext{
1 - Universidade Anhembi Morumbi.

2- Núcleo de Mastologia - Hospital Sírio Libanês
}

\author{
Contato do Autor / Mail to: \\ Bernardo Pinheiro da Senna Nogueira Batista - bernardonb@uol.com.br \\ Rua Professor Atílio Innocenti, 683, Itaim Bibi, São Paulo - SP. CEP: 04538-001.
}




\section{INTRODUÇÃO}

0 câncer de mama é o segundo mais frequente no mundo, sendo o mais frequente entre as mulheres ${ }^{1}$. A cirurgia é o tratamento de escolha, sendo a mastectomia a indicação mais frequente. Mesmo nas cirurgias conservadoras, as sequelas estéticas do tratamento podem causar grande sofrimento às pacientes ${ }^{2}$.

A reconstrução da mama pode ser realizada no mesmo tempo da cirurgia oncológica, quando é chamada de imediata, ou postergada para uma intervenção secundária. As reconstruções mamárias imediatas apresentam benefícios psicológicos e sociais para as pacientes, quando comparadas às reconstruções mamárias tardias ${ }^{3}$, sem reduzir a segurança oncológica ou aumentar os riscos em pacientes bem selecionadas. Uma série de fatores são considerados para a indicação de reconstrução mamária imediata. Idade, presença de comorbidades clínicas, índice de massa corporal (IMC), tabagismo e cirurgia prévia da mama são fatores de risco reconhecidos para complicações pós-operatórias. 0 tipo de tratamento oncológico realizado (ressecção segmentar ou mastectomia, por exemplo) provavelmente influenciará na necessidade de reconstrução mamária, assim como a realização de quimioterapia neoadjuvante 4,5 .

Realizamos um estudo descritivo para identificar se os fatores de risco descritos estão associados à contraindicação de reconstrução mamária imediata em um serviço filantrópico da cidade de São Paulo.

Esse trabalho teve como objetivo identificar se os fatores de risco descritos estão associados à contraindicação de reconstrução mamária imediata em um serviço filantrópico da cidade de São Paulo.

\section{MÉTODOS}

Registros de uma base de dados mantida prospectivamente incluindo todas as pacientes operadas no Serviço de Filantropia do Núcleo de Mastologia do Hospital Sírio Libanês durante o ano de 2015 foi consultado.

Os fatores de risco analisados foram: Idade, comorbidades, tabagismo, índice de massa corporal e tratamento neoadjuvante. A idade foi organizada como uma variável contínua e foi encontrada a média etária das pacientes que realizaram reconstrução imediata das mamas. Foi considerado no estudo apenas a presença ou não de comorbidades. Em relação ao tabagismo, consideramos dois grupos distintos, sendo um composto por pacientes tabagistas e outro por pacientes não tabagistas e ex tabagistas. Para índice de massa corporal foi encontrada a média e o desvio padrão das pacientes que realizaram reconstrução imediata; e para análise de valor foi utilizado o padrão preconizado pela Organização Mundial de Saúde (OMS). O tratamento neoadjuvante foi organizado em dois grupos, sendo um composto pelas pacientes que realizaram o tratamento e outro das que não realizaram. A indicação de reconstrução imediata das mamas foi considerada como desfecho principal.

As variáveis contínuas foram descritas por suas médias e desvios padrão. As distribuições destas variáveis entre os grupos de desfecho foram comparadas através do teste $t$ de Student. As variáveis categóricas foram descritas em valores absolutos e relativos. A associação destas variáveis com a indicação de reconstrução foi realizada pelo teste de chi quadrado. As analises estatísticas foram realizadas com auxilio de software Stata ${ }^{\circledR}$ 14. O nível de significância assumido foi de $5 \%$.

\section{RESULTADOS}

Foram incluídas no estudo 225 pacientes operadas por câncer de mama. Destas, 124 (55,1\%) pacientes realizaram a reconstrução mamária no mesmo tempo cirúrgico. Os resultados do trabalho estão sintetizados na tabela 1.

A média de idade das pacientes que realizaram reconstrução imediata das mamas foi 52,3 com desvio padrão de 1,0. Das pacientes que não realizaram, a média foi 60,7 com desvio padrão de 1,4 . Sendo valor de $p=$ 0,0001 .

Cento e trinta e oito pacientes $(61,3 \%)$ possuíam alguma comorbidade clínica. Dessas, 65 (47\%) realizaram reconstrução imediata, enquanto $73 \quad(52,8 \%)$ não realizaram. 0 valor de $p$ foi 0.002 e qui ${ }^{2}=9,3$.

Das pacientes tabagistas, $16(59,3 \%)$ realizaram resconstrução imediata. Já do grupo de pacientes composto por ex tabagistas e por pacientes que nunca fumaram, 102 (58,7\%) foram submetidas ao procedimento cirúrgico.

A média de IMC das pacientes que realizaram reconstrução imediata das mamas foi 27 com desvio padrão de 0,4. Das pacientes que não realizaram, a média foi 28 com desvio padrão de 0,5. Sendo valor de $p=0,09$.

Das pacientes que realizaram neoadjuvância, 55 (73,3\%), também foram submetidas à reconstrução imediata. Das que não foram submetidas à métodos auxiliares de tratamento oncológico, 68 (45,6\%) fizeram a reconstrução no mesmo tempo cirúrgico.

\section{DISCUSSÃO}

Através do presente estudo, percebemos que a idade teve relevância estatística, pois a média de idade das pacientes que realizaram a reconstrução imediata foi significativamente menor do que as que não realizaram, como já esperado, devido ao aumento de riscos que a idade carrega. 
Tabela 1- Relação entre os fatores de risco analisados e o fato das pacientes terem ou não realizado reconstrução imediata das mamas.

\begin{tabular}{|c|c|c|c|c|c|}
\hline \multirow[b]{2}{*}{ Faixa etâria } & \multicolumn{2}{|c|}{$\begin{array}{c}\text { Năo realizou reconstrução } \\
\text { imediata } n=101\end{array}$} & \multicolumn{2}{|c|}{$\begin{array}{c}\text { Realizou reconstrução } \\
\text { imediata } n=124\end{array}$} & \multirow[t]{2}{*}{$\mathbf{P}$} \\
\hline & Mêdia & DP & Mêdia & DP & \\
\hline Idade & 60,7 & 1,4 & 52,3 & 1 & 0,0001 \\
\hline Comorbidades & $\mathbf{N}$ & $\%$ & $\mathrm{~N}$ & $\%$ & \\
\hline $\begin{array}{l}\text { Não possui } \\
\text { Possui }\end{array}$ & $\begin{array}{l}28 \\
73\end{array}$ & $\begin{array}{l}32,20 \% \\
52,90 \%\end{array}$ & $\begin{array}{l}59 \\
65\end{array}$ & $\begin{array}{l}67,80 \% \\
47,10 \%\end{array}$ & 0,002 \\
\hline Tabagismo & $\mathbf{N}$ & $\%$ & $\mathbf{N}$ & $\%$ & \\
\hline $\begin{array}{c}\text { Tabagista } \\
\text { Ex tabagista + nunca fumou }\end{array}$ & $\begin{array}{l}11 \\
72\end{array}$ & $\begin{array}{l}40,70 \% \\
41,30 \%\end{array}$ & $\begin{array}{r}16 \\
102\end{array}$ & $\begin{array}{l}59,30 \% \\
58,70 \%\end{array}$ & 0,95 \\
\hline IMC & Mêdia & DP & Média & DP & \\
\hline Îndice de massa corporal & 28,2 & 0,5 & 27 & 0,4 & 0.09 \\
\hline Tratamento neoadjuvante & $\mathbf{N}$ & $\%$ & $\mathbf{N}$ & $\%$ & \\
\hline $\begin{array}{l}\text { Não realizou } \\
\text { Realizou }\end{array}$ & $\begin{array}{l}81 \\
20\end{array}$ & $\begin{array}{l}54,30 \% \\
26,60 \%\end{array}$ & $\begin{array}{l}68 \\
55\end{array}$ & $\begin{array}{l}45,60 \% \\
73,30 \%\end{array}$ & 0 \\
\hline
\end{tabular}

A presença de comorbidades apresentou relação estatística com a indicação de cirurgia plástica imediata. Viu-se que a maioria das pacientes que não apresentavam comorbidades realizou cirurgia para reconstrução plástica no mesmo tempo cirúrgico da cirurgia oncológica, enquanto as pacientes com comorbidades, em sua maioria, deixaram a cirurgia plástica para um segundo tempo, apesar dessa diferença ter sido menor do que no grupo anterior.

Quanto ao tabagismo, ao contrário do que se pensaria, apesar dos conhecidos malefícios que o cigarro causa, incluindo a piora da oxigenação de tecidos, dificultando assim a cicatrização, no caso em questão, esse fator não foi determinante na decisão de fazer reconstrução imediata por parte do cirurgião.

o Índice de Massa Corporal não se relaciona estatisticamente com a indicação de reconstrução imediata das mamas. Apesar de intuitivamente considerar a obesidade como fator de risco isolado, percebeu-se que o IMC não é fator de risco independente para complicação pós-operatória6.

Em relação à neodjuvância houve relação estatística. Tal fato sugere que pensando em um estado oncológico provavelmente com maior segurança, infere-se que há menor probabilidade de intervenções futuras, estando o resultado estético final preservado.

No que se refere à indicação de cirurgia eletiva em geral, encontra-se na literatura diversas discussões sobre as condições ideais que o paciente precisa, mas percebe-se uma tendência de que a avaliação deve ser global e personalizada, não apegando-se a valores exatos de exames 5 .

\section{CONCLUSÃO}

A reconstrução de mama imediata traz grandes benefícios para as pacientes, contudo, por meio desse estudo descritivo, percebe-se que existem fatores que auxiliam na decisão do cirurgião plástico no momento de sua indicação. Sendo assim, a indicação cirúrgica deve ser personalizada, levando em consideração diversas características da paciente.

\section{CONFLITOS DE INTERESSE}

Não houve conflitos de interesse.

\section{REFERÊNCIAS}

1. Tipos de câncer [Internet]. Brasil: Instituto Nacional de Câncer José Alencar Gomes da Silva [citado em 15 de janeiro de 2016]. Disponível em: http://www2.inca.gov.br/wps/wcm/connect/tiposdec ancer/site/home/mama/cancer_mama.

2. Saliba, G. A., Carvalho, E. E., Filho, A. F., Alves, J. C., Tavares, M. V., Costa, S. M., et al. (2013). Reconstrução mamária: análise de novas tendências e suas complicações maiores. Revista Brasileira de 
Cirurgia Plástica, Vol. 28(no.4).

3. Oliveira RR, Morais SS, Sarian LO.Efeitos da reconstrução mamária imediata sobre a qualidade de vida de mulheres mastectomizadas. Rev Bras Ginecol Obstet. 2010; 32(12):602-8.

4. Dix P, Howell S - Survey of cancellation rate of hypertensive patients undergoing anaesthesia and elective survey.British Journal of Anaesthesia, 2001;86:789-793.

5. Lorentz, M N; Santos, A X - Hipertensão arterial sistêmica e anestesia -Rev Bras Anestesiol 2005; 55: $5: 586 \quad$ - 594.

6. Fernandes, E. d., Guerra, E. E., Pitrez, F. A., Fernandes, F. M., Rosito, G. B., Gonzáles, H. E., et al. (2010). Avaliação pré-operatória e cuidados em cirurgia eletiva: recomendações baseadas em evidências. Revista da AMRIGS, 54(2): 240-258. 